\title{
Exact Analysis of Effect of Non-uniform Temperature Gradient on Marangoni Convection with Free Slip Condition
}

\author{
S.P. Suma* and Y.H. Gangadharaiah**
}

\begin{abstract}
The effect of non-uniform basic temperature gradient on the onset of Marangoni convection in a horizontal layer with a free-slip bottom heated from below and cooled from above is considered. A linear stability analysis is performed to undertake a detail investigation. The eigenvalues are obtained for lower rigid isothermal and upper free adiabatic boundaries. The resulting eigenvalue problem is solved exactly and single-term Galerkin expansion procedure. The influence of various parameters on the onset of convection has been analyzed. Three non-uniform basic temperature profiles are considered and the results obtained from both the methods are compared and are found to be in good agreement. Some general conclusions about their destabilizing effects are presented.
\end{abstract}

Keywords: Marangoni Convection, Non-Uniform Temperature, free-slip, Galerkin Expansion

\section{Introduction}

An extensive research has been done by many researchers on the onset of convection in a thin layer of fluid heated from below. The

\footnotetext{
* New Horizon College of Engineering, Bangalore 560013; sgchipre@yahoo.com

** gangu.honnappa@gmail.com
}

Received: February 2011; Revised: April 2011 
convective instabilities in such a fluid layer may be due to buoyancy or due to surface tension. The convection driven by buoyancy is known as Rayleigh - Benard convection and that by surface tension is known as Marangoni convection. The onset of Marangoni convection in a layer of fluid with free upper surface and heated from below has been studied extensively because it plays an important in numerous applications such as crystal growth from melts[8], droplet migration in nonuniform temperature fields [12] and flame spreading over a pool of liquid fuel.[10] One of the earliest studies on Marangoni convection was made by Pearson[5] under assumptions of infinitesimally small amplitude analysis with non-deformable free surface and no-slip at the bottom. He showed that thermocapillary forces can cause convection when the Marangoni number exceeds a critical value in the absence of buoyancy forces. Pearson [5] obtained the critical Marangoni number, $M c=79.607$ and the critical wave number ac $=$ 1.9929. His analysis has been extended by many authors. [9], [11], [13]-[15] Linear stability analysis of Marangoni convection with free-slip boundary conditions at the bottom was first treated by Boeck and Thess. [1] For free-slip case, they obtained the critical Marangoni number, $M c=57.598$ and the critical wave number $a c=$ 1.7003 .

Vidal and Acrivos (1966), Debler and Wolf [2] and Nield [4] studied the effect of a non-uniform temperature gradient on the onset of Marangoni convection. Rudraiah [6] and Friedrich and Rudraiah [3] have examined the combined effect of rotation and non-uniform basic temperature gradient on Marangoni convection. They showed that the rotation suppresses convection. The combined effect of non-uniform temperature gradient and magnetic field on Marangoni convection has been studied by Rudraiah et al. [7] Using the single-term Galerkin expansion procedure, they showed that a suitable non-uniform temperature gradient and magnetic field suppress Marangoni convection. The above literature pertains to Marangoni convection subject to a nonuniform basic temperature gradient with no-slip condition.

Here in this paper, we study the effect of non-uniform temperature gradient on Marangoni convection with free-slip at the bottom. The linear stability theory is applied and the resulting eigenvalue 
problem is solved using exact method and single-term Galerkin expansion procedure.

\section{Problem Formulation}

Consider a horizontal fluid layer of depth $d$ with a free upper surface heated from below and subject to a uniform vertical temperature gradient. The fluid layer is bounded below by a rigid, electrically and thermally-perfect conducting wall and bounded above by a free surface. This free surface is subject to a constant heat flux. The interface has a surface tension; $\sigma$ is assumed to be a linear function of the temperature

$$
\sigma=\sigma_{o}-\sigma_{T}\left(T-T_{o}\right)
$$

where $\sigma_{o}$ is the value of $\sigma$ at temperature $T_{o}$ and the constant $\sigma_{T}$ is positive for most fluids. We use Cartesian coordinates with two horizontal $x$ - and $y$ - axis located at the lower solid boundary and a positive $z$-axis is directed towards the free surface. In the basic state, the velocity $\mathrm{q}$ and the temperature $\mathrm{T}$ have the following solutions

$$
\mathrm{q}=0,-\frac{d}{\Delta T} \frac{d T_{o}}{d z}=f(z),
$$

where $f(z)$ is a non dimensional temperature gradient satisfying the condition

$$
\int_{0}^{1} f(z) d z=1
$$

To investigate the effect of the non-uniform temperature gradient on the Marangoni convection, three types of basic temperature profile are chosen.

(i) $f(z)=1$ represents a linear temperature profile;

(ii) $f(z)=2 z$ represents a parabolic temperature profile;

(iii) $f(z)=2(1-z)$ represents a inverted parabolic temperature profile;

Subject to the Boussinesq approximation, the governing equations for an incompressible electrically conducting fluid are expressed as follows: 
Continuity equation:

$$
\nabla \cdot \vec{q}=0
$$

Momentum equation:

$$
\rho_{o}\left(\frac{\partial \vec{q}}{\partial t}+(q \cdot \nabla) q\right)=-\nabla p+\mu \nabla^{2} \vec{q}
$$

Energy equation:

$$
\frac{\partial T}{\partial t}+(q \cdot \nabla) T=\kappa \nabla^{2} \mathrm{~T}
$$

where $q, T, p, v, k$ and $\eta$ denote the velocity, temperature, pressure, density, kinematic viscosity, thermal diffusivity and electrical resistivity, respectively. When motion occurs, the upper free surface of the layer will be deformable with its position at $z=d+$ $f(x, y, t)$ at the free surface; we have the usual kinematic condition together with the conditions of continuity for the normal and tangential stresses. The temperature obeys the Newton's law of cooling, $\mathrm{k} \frac{\partial T}{\partial n}=h\left(T-T_{\infty}\right)$, where $k$ and $h$ are the thermal conductivity of the fluid and the heat transfer coefficient between the free surface and the air, respectively, and $n$ is the outward unit normal to the free surface. The boundary conditions at the bottom wall, $z=0$, are free-slip and conducting to the temperature perturbations.

To simplify the analysis, it is convenient to write the governing equations and the boundary conditions in a dimensionless form. In the dimensionless formulation, scales for length, velocity, time and temperature gradient are taken to be $\mathrm{d}, \mathrm{k} / \mathrm{d}, \mathrm{d}^{2} / \mathrm{k}$ and $\Delta \mathrm{T} /\left(\mathrm{a} \sqrt{\gamma\left(\Delta \mathrm{Td} / \rho_{0} \mathrm{kv}\right)}\right)$ respectively. Furthermore, two dimensionless groups appearing in the problem are the Marangoni number, $\mathrm{M}=\gamma(\Delta \mathrm{Td} /$ $\rho_{0} \mathrm{kv}$ ) and the Prandlt number, $\mathrm{P}_{\mathrm{r}}=\mathrm{v} / \mathrm{k}$.

\section{Linearized Problem}

We study the linear stability of the basic state by seeking perturbed solutions for any quantity $\Phi(\mathrm{x}, \mathrm{y}, \mathrm{z}, \mathrm{t})$ in terms of normal modes in the form

$$
\Phi(\mathrm{x}, \mathrm{y}, \mathrm{z}, \mathrm{t})=\Phi_{0}(\mathrm{x}, \mathrm{y}, \mathrm{z}, \mathrm{t})+\emptyset(\mathrm{z}) \exp \left[i\left(\alpha_{x} x+\alpha_{y} y+s t\right)\right],
$$


where $\Phi_{0}$ is the value of $\Phi$ in the basic state,$a=\sqrt{\alpha_{x}^{2}+\alpha_{y}^{2}}$ is the total horizontal wave number of the disturbance and $s$ is a complex growth rate with a real part representing the growth rate of the instability and the imaginary part representing its frequency. At marginal stability, the growth rate $s$ of perturbation is zero and the real part of $s, \mathrm{R}(s)>0$ unstable modes while $\mathrm{R}(s)<0$ represents stable modes. Substituting equation (7) into equations (4) - (6) and neglecting terms of the second and higher orders in the perturbations we obtain the corresponding linearized equations involving only the $\mathrm{z}$ dependent parts of the perturbations to the temperature and the $\mathrm{z}$-components of the velocity denoted by $T$ and $w$ respectively,

$$
\begin{aligned}
& \qquad \begin{array}{l}
\left(D^{2}-a^{2}\right)^{2} w=0 \\
\left(D^{2}-a^{2}\right) T=-\mathrm{f}(\mathrm{z}) \mathrm{w}
\end{array} \\
& \text { subjected to } w=0 \\
& \begin{array}{c}
D^{2} w=0 \\
\mathrm{~T}=0
\end{array} \\
& \text { Evaluated on } \mathrm{z}=0, w=0 \\
& D \mathrm{~T}=0 \\
& \quad D^{2} w+M a^{2} \theta=0
\end{aligned}
$$

\section{Method of Solutions}

The resulting eigenvalue problem is solved exactly, in general, with $\mathrm{M}$ as an eigenvalue. Besides, an analytical expression for the critical Marangoni number is also obtained by single-term Rayleigh-Rits technique method with wave number $a$ as a perturbation parameter. 


\subsection{Exact Method}

Since equation (8) is independent of $W$. equation (18) can be directly solved to get the general solution in the form

$$
\mathrm{W}=\mathrm{A} \cosh (\mathrm{az})+\mathrm{B} \sinh (\mathrm{az})+\mathrm{C} \mathrm{z} \cosh (\mathrm{az})+\mathrm{D} \mathrm{z} \sinh (\mathrm{az})
$$

Using the boundary conditions (10) - (15) from equation (8), we obtain the expression for Marangoni number, $\mathrm{M}$ on the marginal curve for three types of basic temperature profile:

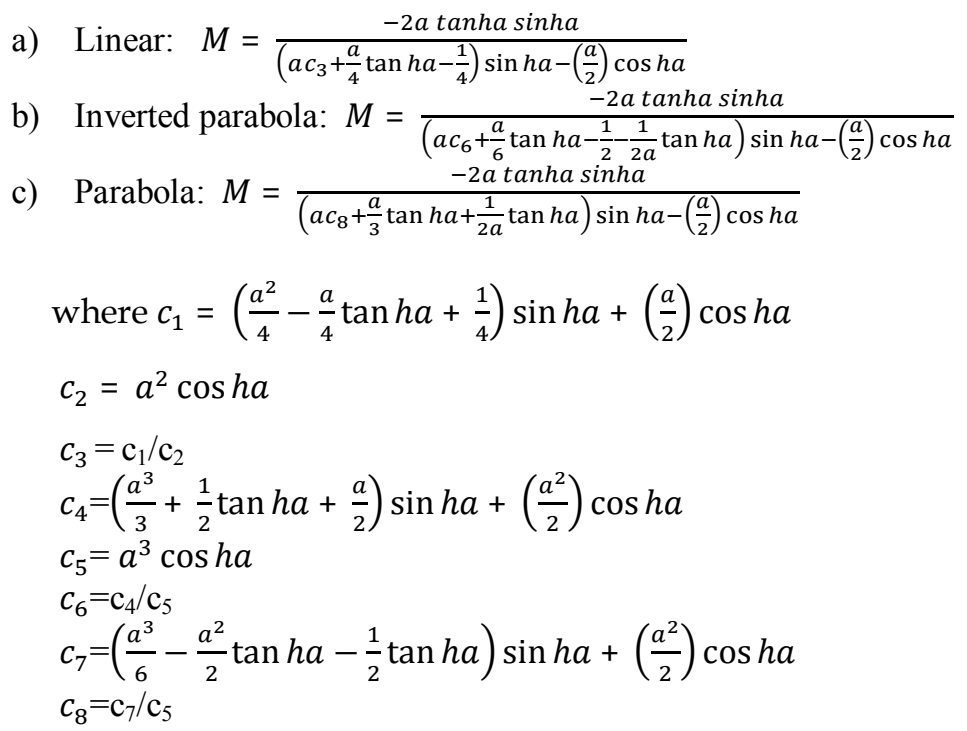

\subsection{Galerkin Method}

The single-term Rayleigh-Rits technique to find the critical eigenvalue. Multiplying equation(8) by $w$ and equation (9) by $T$, integrating the resulting equations by parts with respect to $z$ from 0 to 1 , using the boundary conditions (10) - (15) and taking $\mathrm{w}^{=} \mathrm{Aw}_{1}$, $\mathrm{T}=\mathrm{BT}_{1}$ in which $A$ and $B$ are constants and $w_{1}$ and $\mathrm{T}_{1}$ are trial functions, yields the following equation for the eigenvalue:

$$
\mathrm{M}=\frac{-\left\langle\left(\mathrm{D}^{2} \mathrm{~W}_{1}\right)^{2}+2 \mathrm{a}^{2}\left(\mathrm{DW}_{1}\right)^{2}+\mathrm{a}^{4} \mathrm{~W}_{1}{ }^{2}\right\rangle\left\langle\left(\mathrm{DT}_{1}\right)^{2}+\mathrm{a}^{2} \mathrm{~T}_{1}{ }^{2}\right\rangle}{\mathrm{DW} \mathrm{W}_{1}(1) \mathrm{T}(1) \mathrm{a}^{2}\left\langle\mathrm{~W}_{1} \mathrm{f}(\mathrm{z}) \mathrm{T}_{1}\right\rangle}
$$


In equation (20), $\langle\ldots \ldots\rangle$ denotes integration with respect to $z$ between $\mathrm{z}=0$ and $\mathrm{z}=1$ we select the following trial functions

$$
\mathrm{W}_{1}=z\left(\mathrm{z}^{3}-2 \mathrm{z}^{2}+1\right), \mathrm{T}_{1}=\mathrm{z}(1-\mathrm{z} / 2)
$$

Such that satisfy all the boundary condition (10)-(14) except the boundary condition(15) but the residual from this is included residual from the differential equations. Substituting equations (21) into (20), we get

$$
M=\frac{\left(31 \mathrm{a}^{4}+612 \mathrm{a}^{2}+3024\right)\left(5+2 \mathrm{a}^{2}\right)}{9450 \mathrm{a}^{2}\left\langle\mathrm{z}^{2}\left(\mathrm{z}^{3}-2 \mathrm{z}^{2}+1\right)(1-\mathrm{z} / 2) \mathrm{f}(\mathrm{z})\right\rangle}
$$

From equation (22) we obtain the expression for Marangoni number for three basic temperature profiles:

a) Linear: $M_{1}=\frac{8\left(31 \mathrm{a}^{4}+612 \mathrm{a}^{2}+3024\right)\left(5+2 \mathrm{a}^{2}\right)}{2655 \mathrm{a}^{2}}$

b) Inverted parabola: $M_{2}=\frac{8\left(31 \mathrm{a}^{4}+612 \mathrm{a}^{2}+3024\right)\left(5+2 \mathrm{a}^{2}\right)}{2295 \mathrm{a}^{2}}$

c) Parabola: $M_{3}=\frac{8\left(31 \mathrm{a}^{4}+612 \mathrm{a}^{2}+3024\right)\left(5+2 \mathrm{a}^{2}\right)}{3015 \mathrm{a}^{2}}$

\section{Discussion and Conclusion}

The marginal stability curves in the (a, M) plane are obtained. The region above the marginal stability curve represents unstable modes and the disturbances will grow. The region below the curve represents stable modes and all disturbances will decay. The critical Marangoni number, $M c$ for the onset of steady convection is defined to be the global minimum of the corresponding marginal stability curves. For when $\mathrm{M}<\mathrm{Mc}$, all disturbances are stable while for $M>M c$, a range of unstable disturbances exist.

The effects of a non-uniform temperature gradient on the onset of Marangoni convection in afluid layer with free-slip at the bottom has been studied. The exact method and Galerkin procedure used and the following conclusion may be made from this study. A 
comparison of the critical Marangoni number is shown in Table 1 and the values are found to be in good agreement. Figures 1 and 2 shows that the parabolic function is the most destabilizing basic temperature distribution, and the inverted parabolic is most stabilizing temperature distribution.

\begin{tabular}{|l|l|l|l|}
\hline Temperature Profiles & Mc(Exact method) & Mc (Galerkin method) & Ac \\
\hline Linear & 57.6037 & 56.771 & 1.716 \\
Inverted parabola & 84.3201 & 65.677 & 1.716 \\
Parabola & 43.743 & 49.993 & 1.716 \\
\hline
\end{tabular}

Table 1: Critical Marangoni Number for Different Types of Basic Temperature Profiles

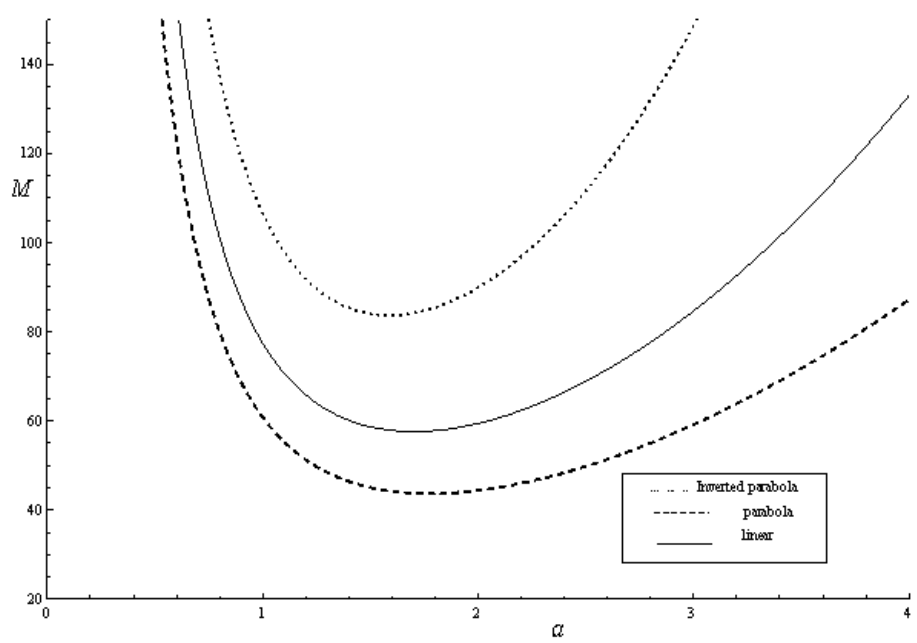

Figure 1: Marginal Stability Curves Plotted for Different Types of Basic temperature Profiles (Exact method) 
Mapana J Sci, 10, 2(2011) Effect of Non-uniform Temperature Gradient

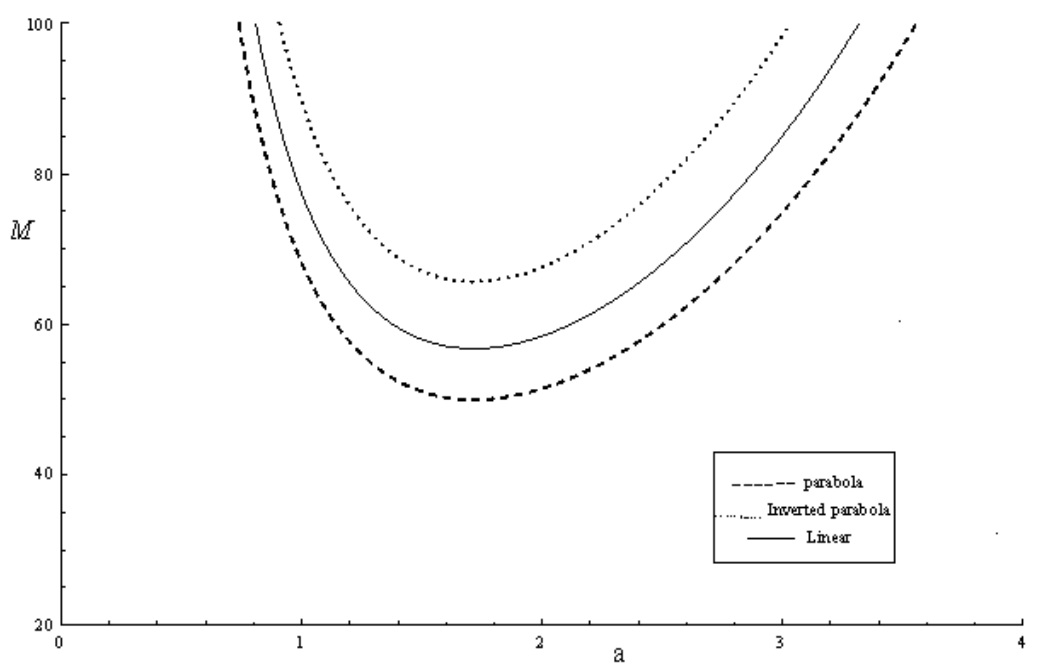

Figure 2: Marginal Stability Curves Plotted for Different Types of Basic temperature Profiles (Galerkin method)

\section{References}

[1] Boeck, T. and Thess, A., 1997. "Inertial Bẻnard-Marangoni Convection", J. Fluid Mech. 350, pp. 149-175.

[2] Debler, W.R. and Wolf, L.F., 1970. "The Effects of Gravity and Surface Tension Gradients on Cellular Convection in Fluid Layers with Parabolic Temperature Profiles", Trans. Am. Soc. Mech. Engrs, Series C, J. Heat Transfer 92, pp. 351-358.

[3] Friedrich, R. and Rudraiah, N., 1984. "Marangoni Convection in a Rotating Fluid Layer with Non-Uniform Temperature Gradient", Int. J. Heat Mass Transfer 27, pp. 443-449.

[4] Nield, D.A., 1975. “The Onset of Transient Convective Instability", J. Fluid Mech. 71, pp. 441-454.

[5] Pearson, J.R.A., 1958. “On Convection Cells Induced by Surface Tension", J. Fluid Mech. 4, pp. 489-500.

[6] Rudraiah, N., 1982. "The Onset of Transient Marangoni Convection in a Liquid Layer Subjected to Rotation about a Vertical Axis", Mater. Sci. Bull. Indian Acad. Sci. 4, pp. 297-316. 
[7] Rudraiah, N., Ramachandramurthy, V. and Chandna, O.P., 1985. "Effects of Magnetic Field and Non- Uniform Temperature Gradient on Marangoni Convection", Int. J. Heat Mass Transfer 28(8), pp. 1621-1624.

[8] Schwabe, D., 1981. "Marangoni Effects in Crystal Growth Melts", PCH Physicochemical Hydrodynamics 2, pp. 263-280.

[9] Scriven, L. E. and Sternling, C.V., 1964. “On Cellular Convection Driven by Surface-Tension Gradients: Effect of Mean Surface Tension and Surface Viscosity", J. Fluid Mech. 19, pp. 321- 340.

[10] Sirignano, W. A., 1972. "A Critical Discussion of Theories of Flame Spread across Solid and Liquid Fuels", Comb. Sci. Tech. 6, pp. 95-105.

[11] Smith, K.A., 1966. “On Convective Instability Induced by SurfaceTension Gradients", J. Fluid Mech. 24, pp. 401-414.

[12] Subramanian, R. S., 1981. "Slow Migration of a Gas Bubble in a Thermal Gradient", A.I.Ch.E.J. 27, pp. 646-654.

[13] Takashima, M., 1970. "Nature of the Neutral State in Convective Instability Induced by Surface Tension and Buoyancy", J. Phys. Soc. Japan. 28, pp. 810.

[14] Takashima, M., 1981a. "Surface Tension Driven Instability in a Horizontal Liquid Layer with a Deformable Free Surface. I. Stationary Convection", J. Phys. Soc. Japan 50, pp. 2745- 2750.

[15] Takashima, M., 1981b. "Surface Tension Driven Instability in a Horizontal Liquid Layer with a Deformable Free Surface. II. Overstability", J. Phys. Soc. Japan 50, pp. 2751-2756. 